\title{
The Wall Shear Stress of a Pulsatile Blood Flow in a Patient Specific Stenotic Right Coronary Artery
}

\author{
Biyue Liu \\ Department of Mathematics Monmouth University West Long Branch, NJ, USA \\ Email: bliu@monmouth.edu
}

Received 2013

\begin{abstract}
A computer simulation of the blood flow in a patient specific atherosclerotic right coronary artery is carried out to study the blood flow pattern and the wall shear stress (WSS) distribution in the artery. Both temporal and special distribution patterns of the WSS of the non-Newtonian blood flow are presented and the regions on the lumen surface where the WSS is constantly lower than $1 \mathrm{~N} / \mathrm{m}^{2}$ are identified.
\end{abstract}

Keywords: Wall Shear Stress; Stenotic Right Coronary Artery; Non-Newtonian

\section{Introduction}

Atherosclerosis is the chief cause of death in the United States. It is a disease which involves complex interactions of many factors. It is widely believed that the wall shear stress (WSS) is one of the important local biomechanical factors responsible for the initiation and the progression of atherosclerosis [1-7]. A detailed hemodynamic evaluation of the spatial and temporal WSS distributions may give additional insight to understanding the progression of the disease and may have useful clinical value.

In the past few decades, numerous clinical researches and computer simulations have been performed to study the flow phenomena in human atherosclerotic arteries and to investigate the correlation between the WSS and the intima-media thickness [1-10]. Caro et al. [1] suggested that the distribution of fatty streaking in human aorta might be coincident with the regions in which the shear rate at the arterial wall is locally reduced. Friedman et al. [2] and Nerem et al. [6] performed experiments that showed an accelerated occurrence of atherosclerosis in human subjects with a coronary geometry. Zarins et al. [7] and Ku et al. [4] estimated shear stress with laserDoppler anemometry and found that intimal thickening bears an inverse relationship to both maximum shear stress and minimum shear stress. Gibson et al. [3] investigated the relationship between the vessel wall shear stress and the rate of atherosclerosis progression by means of quantitative angiography. They found a significant correlation between the low shear stress and an increased rate of atherosclerosis progression. Johnston et al. [10] compared models of Newtonian and Non-Newtonian flow in healthy right coronary arteries with no sign of atheroma. Although the blood flow in stenotic arteries has been extensively studied, there is much to be done both in understanding the disease process itself and also in understanding the role of hemodynamics and the associated WSS as an influence factor on the initiation and the progression of atherosclerosis.

The objectives of the current work are to simulate the blood flow in a stenotic human right coronary artery and to investigate the spatial and temporal WSS distribution patterns during a cardiac cycle.

\section{Mathematical Model}

This study assumes that the fluid is Laminar, Non-Newtonian, viscous and incompressible and the artery wall is rigid. These assumptions have been shown to be adequate for the pulsatile blood flow simulation in the artery models under physiological flow conditions by many investigators. The computational domain is a patient specific atherosclerotic right coronary artery shown in Figure 1(a), re-constructed based on the in vivo intravascular ultrasound (IVUS) image of a patient [9]. The lumen cross section area from the inlet to the outlet is plotted in Figure 1(b), where the horizontal axis is the normalized axial length with inlet $\mathrm{z}=0$ and the outlet $\mathrm{z}=1$. The minimum lumen cross section area at the neck of the stenosis is $0.042 \mathrm{~cm}^{2}$. The maximum area of the cross section proximal to the stenosis is $0.130 \mathrm{~cm}^{2}$. The reduction of the lumen area at the neck of stenosis is approximately $68 \%$. It is a severely stenotic right coronary artery segment.

The time dependent three dimensional Navier Stokes 


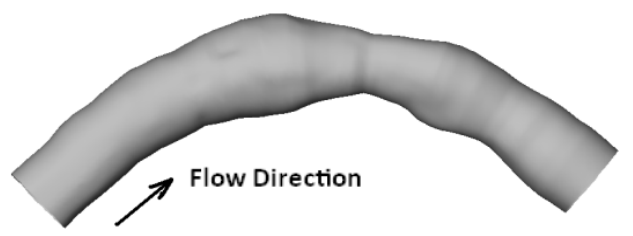

(a)

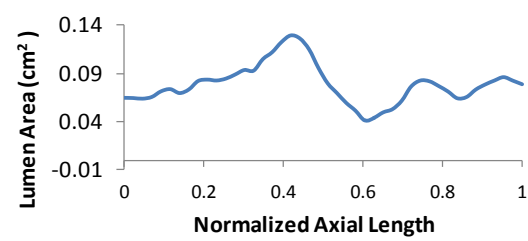

(b)

Figure 1. (a) Geometry of the computational domain; (b) Lumen cross section area from the inlet to the outlet. Neck of the stenosis at $\mathrm{z}=\mathbf{0 . 6}$.

equations are used as the governing equations. The inlet boundary is imposed with a fully developed flow with a physiological human right coronary waveform (Figure 2), scaled to yield a time averaged flow velocity of 0.166 $\mathrm{m} / \mathrm{s}$ at the centre-line. A no-slip condition is applied to the velocities at the wall boundary, treated to be inelastic and impermeable. The outlet boundary is treated as an open boundary with the zero normal stress:

$$
\left(-p \mathbf{I}+\eta\left(\nabla \mathbf{u}+(\nabla \mathbf{u})^{T}\right)\right) \boldsymbol{n}=\mathbf{0}
$$

where $\boldsymbol{n}=\left(n_{1}, n_{2}, n_{3}\right)$ is the outward normal unit vector at the outlet boundary. The initial conditions for the velocity and the pressure are obtained by solving the system of steady state Navier Stokes equations. The blood is treated as a non-Newtonian fluid obeying the Carreau model with the viscosity-shear rate relation:

$$
\eta=\eta_{\infty}+\left(\eta_{0}-\eta_{\infty}\right)\left[1+(\lambda \dot{\gamma})^{2}\right]^{\frac{n-1}{2}}
$$

where $\eta_{0}=0.056 \mathrm{~Pa} \cdot \mathrm{s}$ is the zero shear rate viscosity, $\eta_{\infty}=0.00345 \mathrm{~Pa} \cdot \mathrm{s}$ is the infinite shear rate viscosity, $\lambda=$ $3.313 \mathrm{~s}$ is a parameter, and $n=0.3568$ is a dimensionless parameter $[7,10]$. The blood density $\rho$ is assumed to be constant at $1050 \mathrm{~kg} / \mathrm{m}^{3}$.

\section{Observations and Discussion}

The Navier Stokes equations are solved numerically using the finite element method with piecewise quadratic functions for velocity and piecewise linear functions for pressure over a tetrahedral mesh. Four consecutive cardiac cycles are simulated to ensure that the flow is truly periodic and the computations are repeated over different meshes to ensure that the numerical solutions are mesh independent. The numerical computations are performed using COMSOL 4.2.

Figure 3 presents the contour plots of the WSS $\left(\mathrm{N}^{\mathrm{m}} \mathrm{m}^{2}\right)$

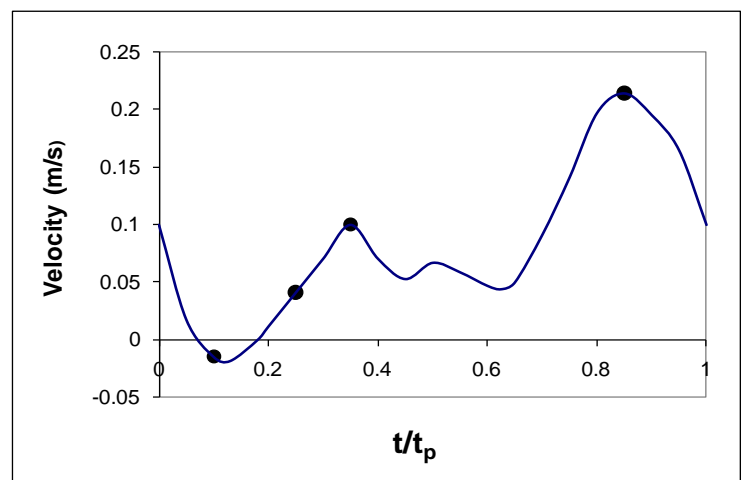

Figure 2. Pulsatile coronary velocity waveform at the inlet.

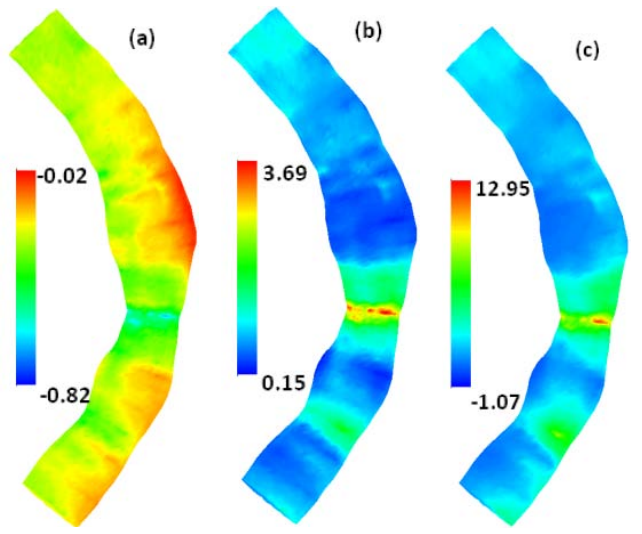

Figure 3. Spatial distribution of the WSS $\left(\mathrm{N} / \mathrm{m}^{2}\right)$ along the artery wall when (a) $t / t_{p}=0.1$, (b) $t / t_{p}=0.35$, (c) $t / t_{p}=0.85$, respectively.

(a) during the deceleration in systole $\left(\mathrm{t} / \mathrm{t}_{\mathrm{p}}=0.1\right)$, (b) at the peak in systole $\left(\mathrm{t} / \mathrm{t}_{\mathrm{p}}=0.35\right)$, and (c) at the maximum flow rate $\left(t / t_{p}=0.85\right)$, respectively. Here the inlet is on the top, and the outlet is on the bottom. Figure 3 shows the spatial distribution pattern of the WSS on the lumen surface. The negative WSS means that the flow moves backwards. The overall negative WSS along the artery wall showing in Figure 3(a) is due to the factor that it is during the reverse flow in the systole (see the first doted point on the velocity waveform in Figure 2). The negative WSS showing in Figure 3(c) indicates that flow recirculation appeared in these regions with a negative WSS. Figures 3(b) and (c) show a basically similar pattern of the spatial distribution of the WSS along the artery at the peaks of the systole and the diastole. The maximum WSS appears at the neck of the stenosis and the WSS is overall high in stenotic region. There is another region with a relatively high WSS downstream where the artery is slightly narrowed. The minimum WSS occurs at the inner wall in the post stenotic region and in the region proximal to the stenosis where the artery expands. The plot of the contour of the WSS at any other time during the forward flow has the same pattern as that showing in Figure 3(b). 
To quantitatively examine the variation of the WSS on the lumen surface, the WSS is averaged on the wall boundary of each lumen cross section and the plots at some representing times are included in Figure $4\left(\mathrm{t} / \mathrm{t}_{\mathrm{p}}=\right.$ $0.1,0.25,0.35$ and 0.85 , respectively, which are doted times in Figure 2). The horizontal axis is the normalized axial length. The inlet is at $\mathrm{z}=0$ and the outlet is at $\mathrm{z}=1$. The neck of the stenosis is at $z=0.6$. Figure 4 confirms the above observation on the special distribution of the WSS from Figure 3. The maximum average WSS occurs at the neck of the stenosis.

Figure 5 plots the WSS in an entire cardiac cycle at various points on the artery wall: (a) at the proximal side of the stenosis and at the neck of the stenosis, (b) in the post stenosis and the downstream. It demonstrates the variation of the local WSS during an entire cardiac cycle at the four locations of most interest: two around the neck of stenosis and two in the post-stenosis region. From Figure 5 we can see that other than the area on the inner wall in the post-stenosis region, the local WSS at any point basically experiences the same fluctuation as that of the flow waveform at the inlet: a dip with negative WSS value caused by the reverse flow in systole followed by two small peaks and a large elevation in diastole. However, the purple curve (labeled PostS) in Figure 5(b) shows that the WSS is constantly low in a whole cardiac cycle on the inner wall in the post stenotic region, without a dominantly high peak when $t / t_{p}=0.85$. These behaviors are consistent with the observation made by the author previously to the simulation based on a simplified geometry with idealized circular cross sections [7].

Many studies [1-5,7,9] have attempted to correlate the WSS with the intimal thickness, suggesting that overall it is in low shear regions that intimal thickness will be greater. Low local velocity, resulting low WSS values, increases residence time and interaction between the blood lipoproteins and vessel endothelium. Long residence time with endothelium results in an increased lipoprotein intake, which might cause the thickening of arterial wall. Clinical studies suggest that intimal thickening

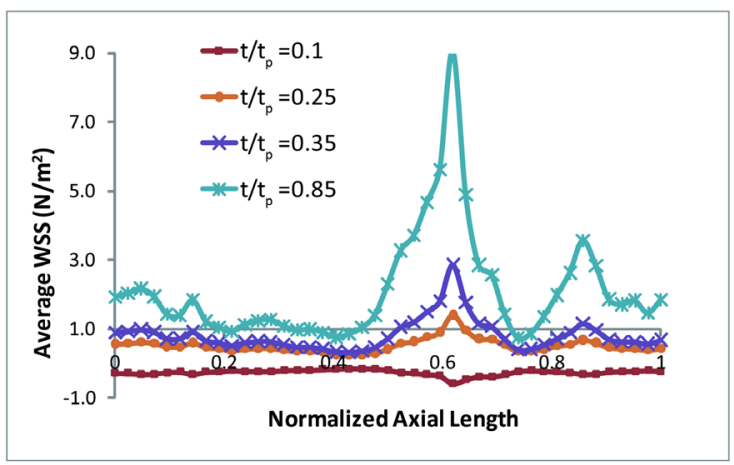

Figure 4. WSS averaged on each lumen cross section when $\mathrm{t} / \mathrm{t}_{\mathrm{p}}=\mathbf{0 . 1}, \mathrm{t} / \mathrm{t}_{\mathrm{p}}=0.25, \mathrm{t} / \mathrm{t}_{\mathrm{p}}=0.35, \mathrm{t} / \mathrm{t}_{\mathrm{p}}=0.85$.
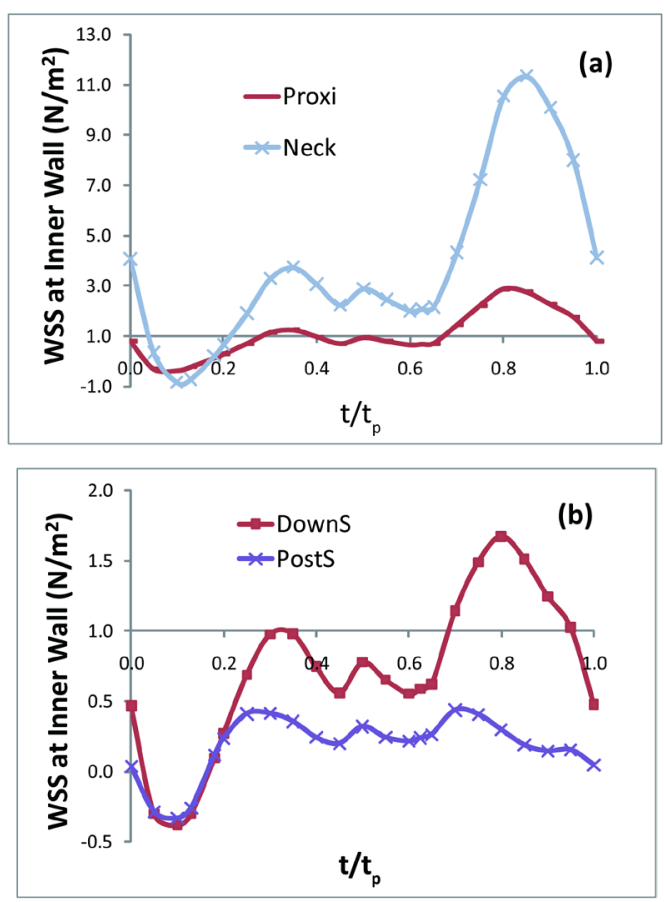

Figure 5. Temporal variation of the WSS at various points on the inner wall (a) at the proximal side of the stenosis and at the neck of the stenosis, (b) in the post stenosis and the downstream of the stenosis.

likely occurs when the average WSS is below $1 \mathrm{~N} / \mathrm{m}^{2}$ (= 10 dynes $/ \mathrm{cm}^{2}$ ), which presents an inverse hyperplasia with respect to the shear stress [5]. Therefore, it is of special interest to know the location and the size of the region on the lumen surface where the WSS is lower than $1 \mathrm{~N} / \mathrm{m}^{2}$ (including the negative WSS resulted from the reverse flow or flow recirculation) and the duration of the low WSS area in a cardiac cycle.

At each point on the lumen surface, we calculated the total length of the time when the location experiences a low WSS $<N / \mathrm{m}^{2}$. Figure 6 presents the contour plot of the duration of the WSS under $N / \mathrm{m}^{2}$ in a cardiac cycle. Figures 6(a)-(c) are the views of the artery from the side, the inner wall and the outer wall, respectively. A point with a value of 1.0 on the lumen surface indicates that the WSS at this location is lower than $1 \mathrm{~N} / \mathrm{m}^{2}$ anytime during the entire cardiac cycle. The accumulated duration time of the WSS lower than $1 \mathrm{~N} / \mathrm{m}^{2}$ in a cycle has the minimum value of 0.17 in the region of stenosis neck. From the length of the interval where the grey curve (labeled Neck) is below the horizontal axis line setting at 1.0 level in Figure 5(a), we can tell that this duration of the low WSS occurs approximately during the systole between $t / t_{p}=0.03$ and $t / t_{p}=0.2$ in a cardiac cycle. It is also apparent that the duration value is 1.0 in the area on the inner wall in the post-stenosis region and the area on the inner wall in the region proximal to the stenosis where the lumen is expanded. This indicates that these 


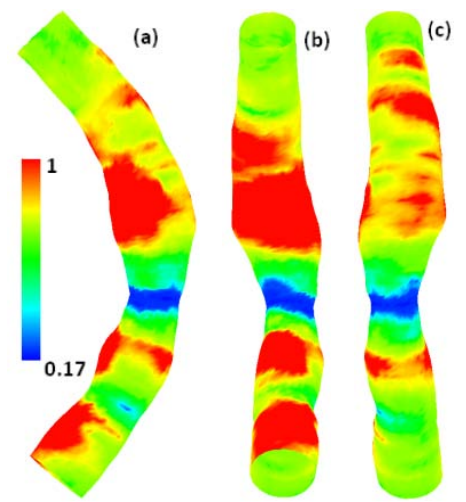

Figure 6. The duration of the WSS under $1 \mathrm{~N} / \mathrm{m}^{2}$ (a) the side view of the artery, (b) the inner wall of the artery, (c) the outer wall of the artery.

areas experience a lower WSS during the whole cardiac cycle. The purple curve (labeled PostS) in Figure 5(b) also demonstrates this, where the whole curve is below the horizontal axis line setting at 1.0 level. Comparing Figures 6(b) with (c) we can see that the low WSS regions mostly occur on the inner wall of the stenotic right coronary artery. The WSS on the outer wall is relatively higher, which may prevent the deposition of the particles and the further intimal thickening along this side of the artery.

\section{Conclusion}

In this work, a computer simulation of the blood flow in a patient specific stenotic right coronary artery has been performed to investigate the phasic variation and the spatial distribution pattern of the wall shear stress on the lumen surface. Based on the computational results presented, we can see that the WSS distribution is highly non-uniform both temporally and spatially. The WSS elevates in the stenotic region, reaches the maximum at the neck of the stenosis, and drops sharply in the post-stenosis region. The areas on the inner wall in the post-stenosis region and in the region proximal to the stenosis are subject to a low WSS less than $N / \mathrm{m}^{2}$ during the entire cardiac cycle.

\section{Acknowledgements}

This work was partially supported by a grant from the Simons Foundation (\#210082 to Biyue Liu) and a sabbatical grant from Monmouth University. The author thanks Dr. Dalin Tang for providing the stenotic right coronary artery data for re-constructing the computational domain.

\section{REFERENCES}

[1] C. G. Caro, J. M. Fitz-Gerald and R. C. Schroter, “Atheroma and Arterial Wall Shear Observation, Correlation and Proposal of a Shear-Dependent Mass Transfer Mechanism for Atherogenesis," Proceedings of the Royal Society B: Biological Sciences, Vol. 177, 1971, pp. 109-159. http://dx.doi.org/10.1098/rspb.1971.0019

[2] M. H. Friedman., G. M. Hutchins, C. B. Bargeron, O. J. Deters and F. F. Mark, "Correlation between Intimal Thickness and Fluid Shear in Human Arteries," Atherosclerosis, Vol. 39, 1981, pp. 425-436. http://dx.doi.org/10.1016/0021-9150(81)90027-7

[3] C. M. Gibson, L. Diaz, K. Kandarpa, F. M. Sacks, R. C. Pasternak, T. Sandor, et al., "Relation of Vessel Wall Shear Stress to Atherosclerosis Progression in Human Coronary Arteries," Arteriosclerosis and Thrombosis, Vol. 13, 1993, pp. 310-315. http://dx.doi.org/10.1161/01.ATV.13.2.310

[4] D. N. Ku, D. P. Giddens, C. K. Zarins, et al, "Pulsatile Flow and Atherosclerosis in the Human Carotid Bifurcation: Positive Correlation between Plaque Location and Low and Oscillating Stress,” Arteriosclerosis, Vol. 5, 1985, pp. 292-302.

[5] D. N. Ku, "Blood Flow in Arteries," The Annual Review of Fluid Mechanics, Vol. 29, 1997, pp. 399-434. http://dx.doi.org/10.1146/annurev.fluid.29.1.399

[6] R. M. Nerem and M. J. Levesque, "The Case for Fluid Dynamics as a Localizing Factor in Atherogenesis,” In: G. Schettler, R. M. Nerem, H. Schimid-Schronbein, H. Mori and C. Diehm, Eds., Fluid Dynamics as a Localizing Factor for Atherosclerosis, Springer-Verlag, Heidelberg, 1983, pp. 26-37. http://dx.doi.org/10.1007/978-3-642-69085-3_4

[7] C. K. Zarins, D. P. Giddens, B. K. Bharadvaj, V. S. Sottiurai, R. F. Mabon and S. Glagov, "Carotid Bifurcation Atherosclerosis: Quantitative Correlation of Plaque Localization with Flow Velocity Profiles and Wall Shear Stress,' Circulation Research, Vol. 53, 1983, pp. 502-514. http://dx.doi.org/10.1161/01.RES.53.4.502

[8] B. Liu and D. Tang, "Influence of Non-Newtonian Properties of Blood on the Wall Shear Stress in Human Atherosclerotic Right Coronary Arteries,” Molecular \& Cellular Biomechanics, Vol. 8, No. 1, 2011, pp. 73-90.

[9] C. Yang, R. Bach, J. Zheng, I. El Naqa, P. K. Woodard, Z. Teng, K. Billiar and D. Tang, "In Vivo IVUS-Based 3D Fluid Structure Interaction Models with Cyclic Bending and Anisotropic Vessel Properties for Human Atherosclerotic Coronary Plaque Mechanical Analysis," IEEE Transactions on Biomedical Engineering, Vol. 56, No. 10, 2009, pp. 2420-2428. http://dx.doi.org/10.1109/TBME.2009.2025658

[10] B. M. Johnston, P. R. Johnston, S. Corney and D. Kilpatrick, "Non-Newtonian Blood Flow in Human Right Coronary Arteries: Transient Simulations," Journal of Biomechanism, Vol. 39, 2005, pp. 1116-1128. http://dx.doi.org/10.1016/j.jbiomech.2005.01.034 